\title{
SNPP VIIRS spectral bands co-registration and spatial response characterization
}

\author{
Guoqing Lin ${ }^{*, \mathrm{a}, \mathrm{b}}$, James C. Tilton ${ }^{\mathrm{b}}$, Robert E. Wolfe ${ }^{\mathrm{b}}$, Krishna P. Tewari ${ }^{\mathrm{a}}$, Masahiro Nishihama ${ }^{\mathrm{c}}$ \\ ${ }^{a}$ Innovim, 7474 Greenway center Dr. Suite 800, Greenbelt, MD, USA 20770; \\ ${ }^{\mathrm{b}}$ NASA Goddard Space Flight Center, Code 619, 8800 Greenbelt Rd, Greenbelt, MD, USA 20771; \\ 'Sigma Space Corporation, 4600 Forbes Blvd, Lanham, MD, USA 20706
}

\begin{abstract}
The Visible Infrared Imager Radiometer Suite (VIIRS) instrument onboard the Suomi National Polar-orbiting Partnership (SNPP) satellite was launched on 28 October 2011. The VIIRS has 5 imagery spectral bands (I-bands), 16 moderate resolution spectral bands (M-bands) and a panchromatic day/night band (DNB). Performance of the VIIRS spatial response and band-to-band co-registration (BBR) was measured through intensive pre-launch tests. These measurements were made in the non-aggregated zones near the start (or end) of scan for the I-bands and M-bands and for a limited number of aggregation modes for the DNB in order to test requirement compliance. This paper presents results based on a recently re-processed pre-launch test data. Sensor (detector) spatial impulse responses in the scan direction are parameterized in terms of ground dynamic field of view (GDFOV), horizontal spatial resolution (HSR), modulation transfer function (MTF), ensquared energy (EE) and integrated out-of-pixel (IOOP) spatial response. Results are presented for the non-aggregation, 2-sample and 3-sample aggregation zones for the I-bands and M-bands, and for a limited number of aggregation modes for the DNB. On-orbit GDFOVs measured for the 5 I-bands in the scan direction using a straight bridge are also presented. Band-to-band co-registration (BBR) is quantified using the prelaunch measured band-to-band offsets. These offsets may be expressed as fractions of horizontal sampling intervals (HSIs), detector spatial response parameters GDFOV or HSR. BBR bases on HSIs in the non-aggregation, 2-sample and 3-sample aggregation zones are presented. BBR matrices based on scan direction GDFOV and HSR are compared to the BBR matrix based on HSI in the non-aggregation zone. We demonstrate that BBR based on GDFOV is a better representation of footprint overlap and so this definition should be used in BBR requirement specifications. We propose that HSR not be used as the primary image quality indicator, since we show that it is neither an adequate representation of the size of sensor spatial response nor an adequate measure of imaging quality.
\end{abstract}

Keywords: Suomi NPP, VIIRS, band-to-band co-registration, ground dynamic field of view, spatial response, ensquared energy, integrated out-of-pixel spatial response, day/night band (DNB).

\section{INTRODUCTION}

The NASA/NOAA Visible Infrared Imager Radiometer Suite (VIIRS) instrument onboard the Suomi National Polar-orbiting Partnership (SNPP) satellite was launched on 28 October 2011. SNPP was put under management of the NASA acquired and NOAA managed Joint Polar Satellite System (JPSS) after restructuring of the National Polarorbiting Environmental Satellite System (NPOESS) and it was renamed from the NPOESS Preparatory Project (NPP) after its successful launch and check out in early $2012^{[1]}$. Copies of VIIRS instrument will be on the follow-on NOAA Joint Polar Satellite System (JPSS) in an afternoon orbit (1:30 PM local time of the ascending node crossing the equator). As a payload originally developed for the NPOESS, the VIIRS instrument inherits the triple-purpose (civilian, military and science applications) design ${ }^{[2,3]}$ that balances requirements such as fine imagery resolution, low noise and broad spectral coverage.

*gary.lin@nasa.gov; glin@innovim.com; phone 301-614-5451; fax 301-614-5269; innovim.com

Earth Observing Systems XVIII, edited by James J. Butler, Xiaoxiong (Jack) Xiong, Xingfa Gu, Proc. of SPIE Vol. 8866, 88661G · (C) 2013 SPIE · CCC code: 0277-786X/13/\$18 · doi: 10.1117/12.2023367 
VIIRS has 5 imagery resolution bands (bands I1 to I5) with 32 detectors each, 16 moderate resolution bands (bands M1 to M16) and a panchromatic day-night band (DNB) with 16 detectors each. The spectral band M16 has two physical bands M16A and M16B that are time delay integrated spatially but tested individually pre-launch on the ground. DNB has three gain stages in four charge-coupled device (CCD) arrays: high gain A-side (HGA), high gain B-side (HGB), mid-gain stage (MGS) and low-gain stage (LGS). These gain stages are selected in on-orbit operations but tested individually pre-launch. These bands collect energy from the earth in a cross-track (scan) direction through a rotating telescope assembly (RTA) and a half angle mirror (HAM) that effectively de-rotates the rays from the RTA ${ }^{[4,5]}$. As the satellite circles the earth in the near-polar orbit and the RTA scans across the track, VIIRS sweep a swath of $\sim 3,000 \mathrm{~km}$ of the earth in a field of regard of $+/-56^{\circ}$. The M-bands have a nominal horizontal sampling interval (HSI) of $750 \mathrm{~m}$ at nadir, the I-bands have a nominal HSI of $375 \mathrm{~m}$ at nadir, and the day-night band (DNB) has a near-constant nominal 750 m HSI throughout the scan. Due to the effects of slant distance and earth curvature, the HSI from each detector grows from nadir to the edges of scan and ground coverage overlaps more and more towards the edge of scan ("bowtie" effect). An along-scan aggregation scheme is employed to limit the growth of pixels in the scan direction. In the nadir region of the scan, three samples are aggregated (called 3x1 aggregation or Agg3x1). No aggregation (called 1x1 aggregation or Agg1x1) is performed near the edges of the scan and two samples are aggregated (called 2x1 aggregation or Agg2x1) in the middle of the scan. Also, a bow-tie deletion scheme is applied to trim readings of one (two) and two (four) M (I) band detectors at the edges of every band detector array in the Agg2x1 and Agg1x1 zones, respectively. For the DNB, 32 distinctive sample aggregation and bow-tie "compression" (contiguous CCD detectors selected to form 16 "super" detectors close to the center in the track direction) is used to keep the ground sampling interval near constant throughout the scan with Aggregation Mode 1 at nadir and Aggregation Mode 32 at the edges of $\operatorname{scan}^{[1,6,7]}$. Performance of spatial response and band-to-band co-registration (BBR) was measured through intensive prelaunch tests and the results have been presented in terms of requirement compliance ${ }^{[4,5,8]}$.

This paper presents results after re-processing the pre-launch data from thermal vacuum tests at the nominal temperature performance plateau. These ground tests were set in the diagnostic mode, meaning non-aggregation for all detectors for the I-bands and M-bands. Line spread functions (LSFs) and band-to-band offsets in the Agg2x1 and Agg3x1 zones are constructed analytically from the non-aggregation test data. For the DNB spatial responses, aggregation modes 18, 22 and 31 were tested for all four CCD arrays. Section 2 presents the along scan LSF parameters in terms of dynamic field of view (DFOV), horizontal spatial resolution (HSR), modulation transfer function (MTF), ensquared energy (EE) and integrated out-of-pixel (IOOP) spatial responses. Results are presented for the three zones for the I-bands and M-bands, and for the three aggregation modes for the DNB. On-orbit GDFOVs for the 5 I-bands in the scan direction using a straight bridge are also presented in this Section. Section 3 presents the I-band and M-band band-to-band co-registration (BBR) matrices based on HSIs in the three aggregation zones. BBR matrices based on scan direction GDFOV and HSR are compared to the BBR matrix based on HSI in the non-aggregation zone in this Section as well. Concluding remarks are presented in the last Section.

\section{VIIRS SENSOR SPATIAL RESPONSES}

The VIIRS sensor spatial impulse responses were measured for every detector before launch. Since the detectors were fabricated in an optically apparent rectangular shape, their full impulse responses were determined by combining two orthogonal line spread functions (LSFs) measurements. These LSFs were measured during the thermal vacuum (TVac) testing. The tests included LSFs measurements in the scan and track directions at the cold and nominal performance temperature plateaus, and some special tests at the hot performance plateau. The scan direction LSFs presented here are from the test at the nominal performance temperature plateau, representing the nominal operating conditions in space. The LSFs in the track direction are mostly squares ${ }^{[4,8]}$, with their width nearly the same as the horizontal sampling interval (HSI).

The ground tests were performed in the diagnostic mode, meaning none of the detectors were aggregated. Band M16 is time delay integrated from bands M16A and M16B, but only band M16B was measured and included in this paper. The LSFs in the Agg2x1 and Agg3x1 zones are derived from the LSFs in the Agg1x1 zone. Angular sampling intervals (ASIs) in the scan direction are constants for I-bands and M-bands, respectively at 156 and $312 \mu$ radians in the Agg1x1 zone. The ASIs in the Agg2x1 and Agg3x1 zones are respectively two and three times as large as those in the Agg1x1 zone. In the track direction, ASIs are constants throughout the scan, at 445.5 and $891 \mu$ radians for I-bands and M-bands, respectively ${ }^{[4]}$. For DNB, the track and scan ASIs varies with scan angle in 32 aggregation modes, from $894 \mu$ radians 
for the aggregation mode 1 at nadir to 149 rradians for the aggregation mode 32 at the edges of the scan. The HSIs are projections of ASIs on the ground. It is sometimes referred to as ground sample distance (GSD) or ground sample interval $(\mathrm{GSI})^{[9]}$. They vary with satellite altitude and scan angle. For SNPP, the variation of altitude is tightly controlled by drag make-up maneuvers to maintain 101.5 minutes orbital period and 16 -day repeat cycle ${ }^{[7]}$. Over the equator, the altitude is within $1 \mathrm{~km}$ of $829.8 \mathrm{~km}$. However, the altitude is about $840 \mathrm{~km}$ over the north pole and about 855 over the south pole ${ }^{[5,6]}$. At the mean altitude of $840 \mathrm{~km}$, the post-aggregation HSIs for M-bands are $786 \mathrm{~m}$ and 742 $\mathrm{m}$ in the scan and track direction at nadir, respectively, and $1711 \mathrm{~m}$ and $1658 \mathrm{~m}$ at the edges of scan. For I-bands, the HSIs are twice as finer as the HSIs for the M-bands. For the DNB at this mean altitude, HSIs are maintained within 7\% of $770 \mathrm{~m}$ in the scan direction and within $3 \%$ of $765 \mathrm{~m}$ in the track direction throughout the scan.

Figure 1 depicts four examples of scan direction LSFs. The LSFs for band I3 are typical for the 5 I-bands and the LSFs for band M6 are typical for the 16 M-bands. The M-band exception is band M11 which has a side-lobe about 5\% the magnitude of the main lobe about 2.3 sampling intervals away in the non-aggregated zone. The dashed lines in the subplots for I3 and M6 represent the reference LSFs assuming the detector size and integration drag are equivalent to ASI without optical blur. LSFs for the DNB are mostly squares, with the effects of optical blur on the LSFs near the edges of scan. The two dashed lines represent LSFs in aggregation modes 18 and 31without optical blur.
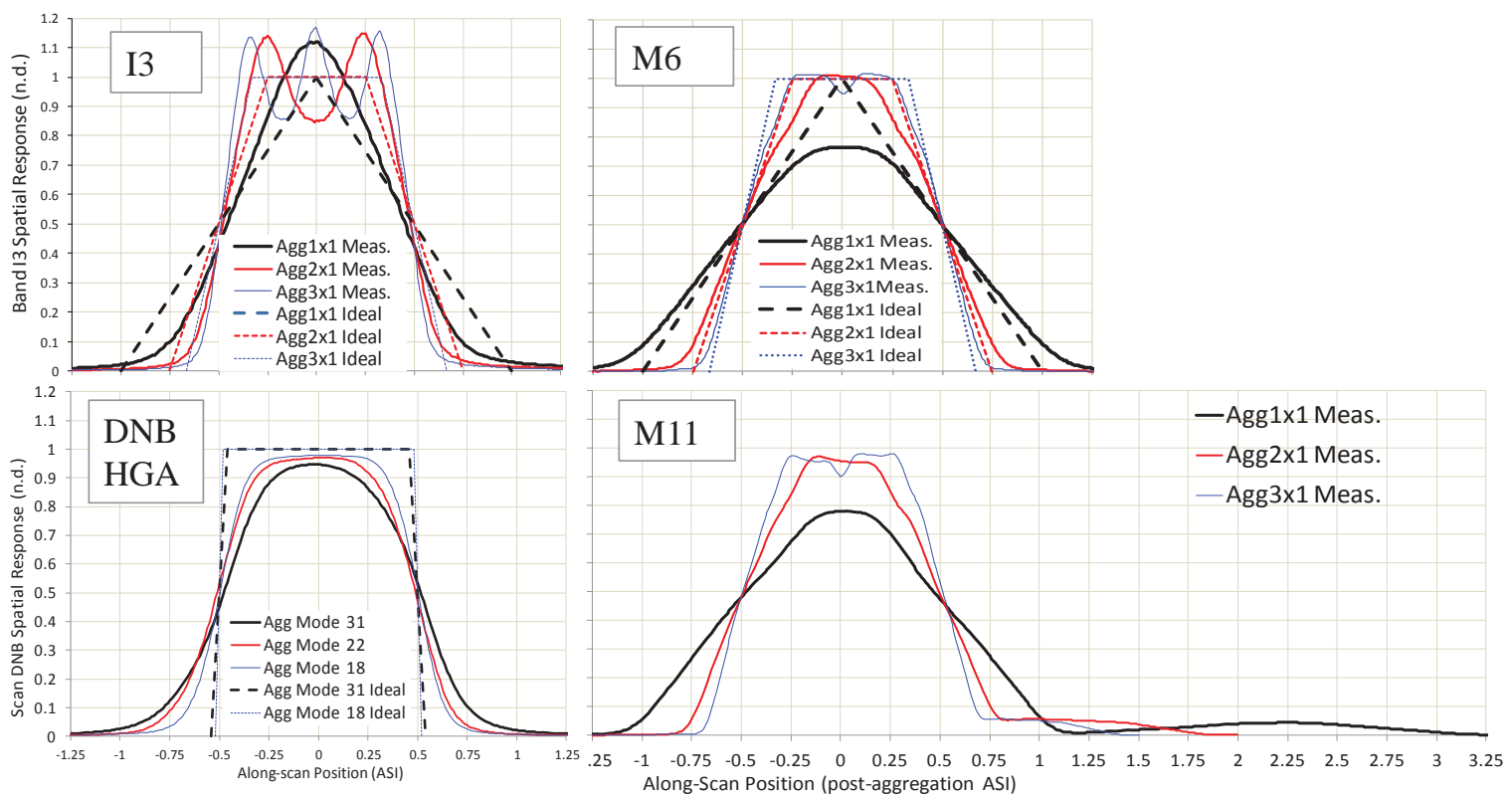

Figure 1. Typical line spread functions (LSFs) for I-bands (represented by I3 here) and M-bands (represented by M6 here). LSFs in Agg2x1 and Agg3x1 zones are derived from LSFs in non-aggregated zones from ground testing. DNB LSFs are from HGA only for this paper.

The LSFs in the scan direction may be parameterized in dynamic field of view (DFOV) and horizontal spatial resolution (HSR). DFOV is the full-width at half maximum (FWHM) of the LSF in the scan direction including the effect of time integration. It may be represented in term of ASI. The projection of DFOV on the ground is the ground DFOV (GDFOV). HSR is the half wavelength on the ground where modulation transfer function (MTF) of LSF drops to $1 / 2$ as expressed in Equation (3) below. It is sometimes regarded as "effective" detector (or sensor) resolution and may be expressed in terms of HSI. MTF is a normalized Fourier transfer function commonly used to quantify image quality, i.e.,

$$
\operatorname{MTF}(\omega)=\frac{\mid \text { FourierTansform }(L S F) \mid}{\mid \text { FourierTansform }\left.(L S F)\right|_{\mid \omega=0}},
$$

where $\omega$ is expressed in fraction of Nyquist frequency that is the maximal detectible sinusoidal waves by the Nyquist sampling theorem ${ }^{[10]}$, i.e., 


$$
f_{\text {Nyquist }}=\left(\frac{1}{2 H S I}\right) .
$$

From the MTF curve, the HSR may be calculated as

$$
\operatorname{MTF}\left(\frac{1}{2 H S R}\right)=0.5 .
$$

From the LSF, we may also calculate the ensquared energy ${ }^{[11]}$,

$$
E E=\int_{|x| \leq \frac{1}{2}} \operatorname{LSF}(x) d x
$$

which represents the total weight within the ASI. Integrated out-of-pixel (IOOP) spatial response may also be introduced to represent the total weight contaminated by the neighboring pixels, i.e.,

$$
I O O P=\int_{|x| \succ \frac{1}{2}} \operatorname{LSF}(x) d x=1-E E .
$$

To simplify these calculations of the scan direction EE and IOOP, we assume that the LSFs in the track direction are uniformly distributed within the sampled pixel (i.e., track EE $=1$ and IOOP $=0$ ).

For example, an ideal triangular LSF with a FWHM the same as its ASI, the HSR is 1.13 HSI, MTF is 0.40 at the Nyquist frequency, EE is $75 \%$ and IOOP is $25 \%$. For an ideal square LSF with a FWHM the same as its ASI, the HSR is $0.83 \mathrm{HSI}, \mathrm{MTF}$ is 0.63 at the Nyquist frequency, EE is $100 \%$ and IOOP is $0 \%$.

\subsection{Sensor (detector) Resolutions}

Figure 2 shows the scan direction GDFOV to HSI ratio for each detector for the I-bands and M-bands in the three aggregation zones, as well as for the three DNB (HGA) aggregation modes (31, 22 and 18). For all 5 I-bands, pixels are under sampled at the top of atmosphere. The under-sampling is most acute for bands I3 to I5 in the Agg1x1 zones and may cause aliasing ${ }^{[9]}$. However, when they measure radiance from the ground surface, atmospheric modulation ${ }^{[12]}$ may reduce the under-sampling effect as the optical path lengthens in the Agg1x1 zones, which are the furthest from nadir. For M-bands, pixels in the Agg1x1 zones are over-sampled. However, the pixels in the Agg2x1 and Agg3x1 zones are sampled at the Nyquist frequency. This Nyquist frequency sampling is also true for DNB aggregation modes 31, 22, and 18, and may be extrapolated to all DNB aggregation modes throughout the scan.

Figure 3 shows the scan direction HSR to HSI ratio for each detector for the I-bands and M-bands in the three aggregation zones, and the three DNB (HGA) aggregation modes. By comparing Figures 2 and 3, we can see that for Ibands and M-bands in the Agg1x1 zones, the sensor HSR/HSI ratio is larger than the GDFOV/HSI ratio. However, as the aggregation zones approach nadir with more samples aggregated, the LSF becomes more square-like and GDFOV approaches 1.0 HSI, but HSR approaches 0.83 HSI. This is also true for DNB. Thus, the HSR for a square-like LSF may mis-represent "effective" sensor resolution as the footprint on the ground. In general, GDFOV is a better parameter representing sensor footprint, even though long tails and side- lobes, such as the one for band M11, may not be well represented by GDFOV. 


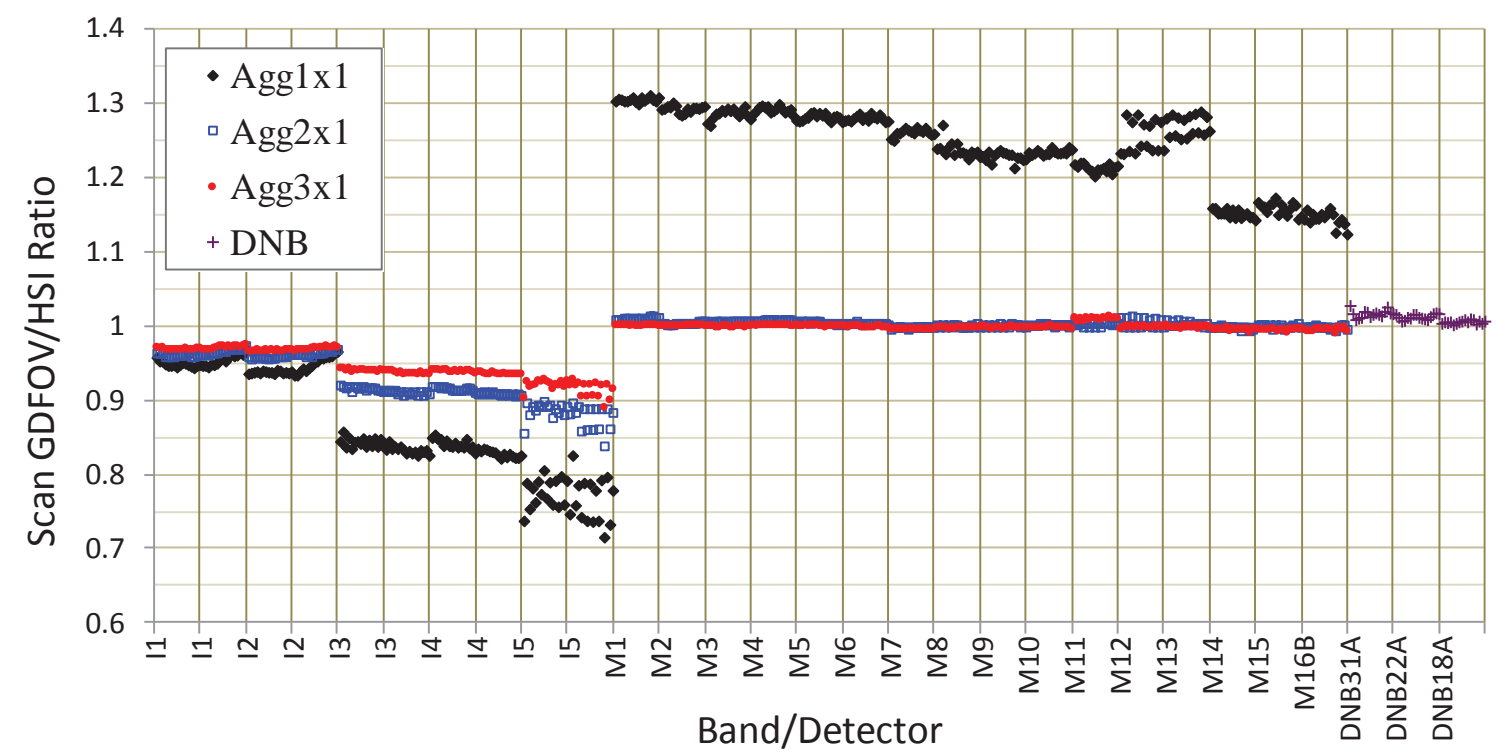

Figure 2. Scan direction GDFOV to HSI ratio for each detector for the I-bands, M-bands and DNB (HGA).

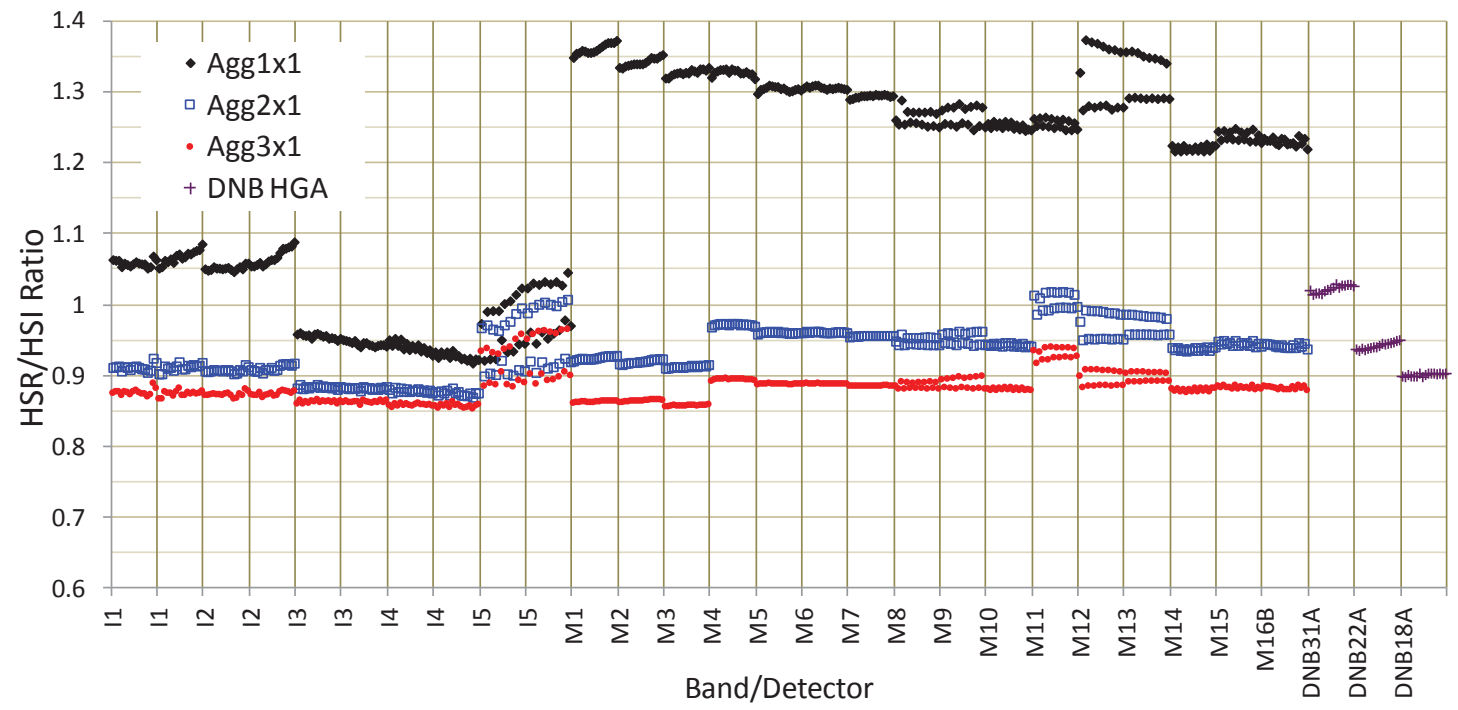

Figure 3. Scan direction HSR to HSI ratio for the each detector for the I-bands, M-bands and DNB (HGA).

\subsection{Imaging Quality Parameters}

Image quality is commonly measured by modulation transfer function (MTF), a measure of contrast reduction of sinusoidal functions. Figure 4 shows the scan direction MTF for each detector for the I-bands and M-bands in the three aggregation zones at 1.0 and 0.5 Nyqusit frequencies. It also shows DNB (HGA) in three aggregation modes, which has values comparable to those for bands I1 and I2. The MTF for M-bands has lower values, especially for the visible bands M1 to M6. The effect of side-lobe in M11 LSF does not show up in the MTF at the 1.0 Nyquist frequency in the nonaggregation zones but does so at the 0.5 Nyquist frequencies. This demonstrates the need to quantify MTF at multiple frequencies.

Figure 5 shows scan direction ensquared energy (EE) for each detector for the I-bands and M-bands in the three aggregation zones, and three DNB (HGA) aggregation modes. I-bands collect more energy within the sampled pixels than M-bands. As aggregation zone approaches nadir, more energy is collected within the sampled pixel. It may be inferred that DNB collects 100\% energy within the sampled pixels as the aggregation modes approaches nadir (aggregation mode 1). 


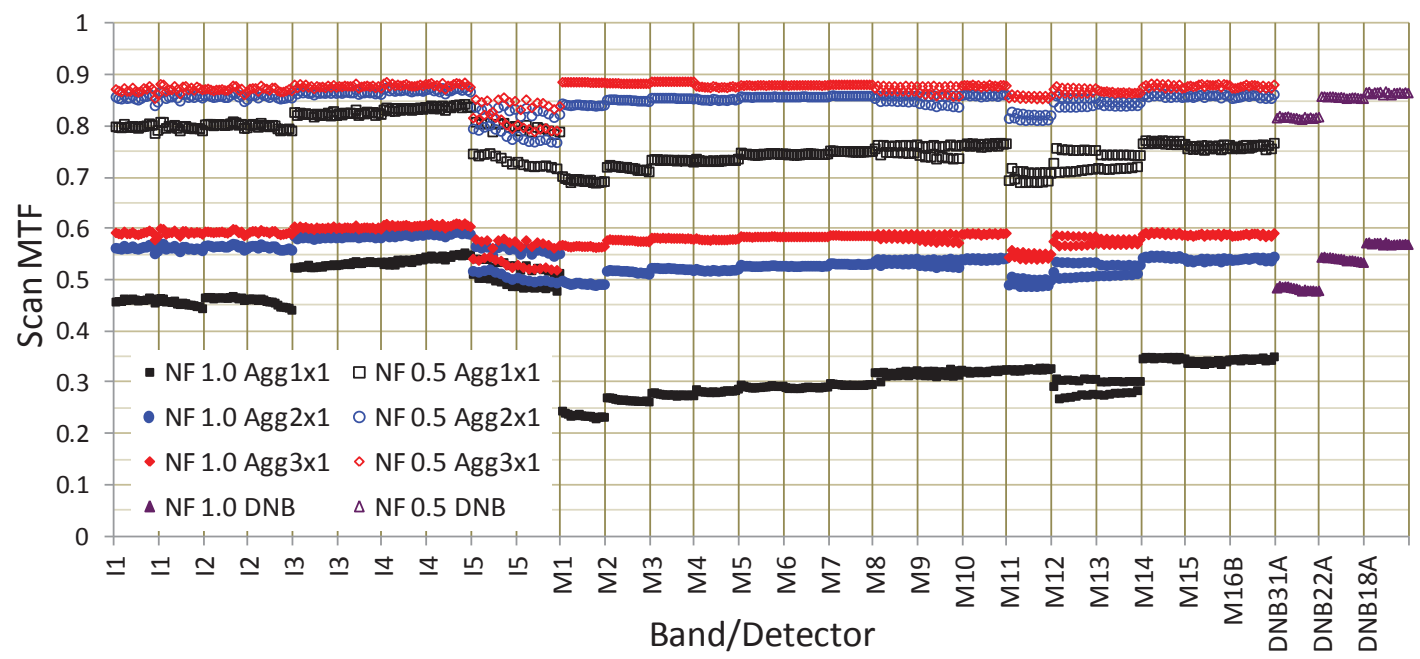

Figure 4. Scan direction MTF for the each detector for the I-bands, M-bands and DNB (HGA) at the 1.0 and 0.5 Nyquist frequencies.

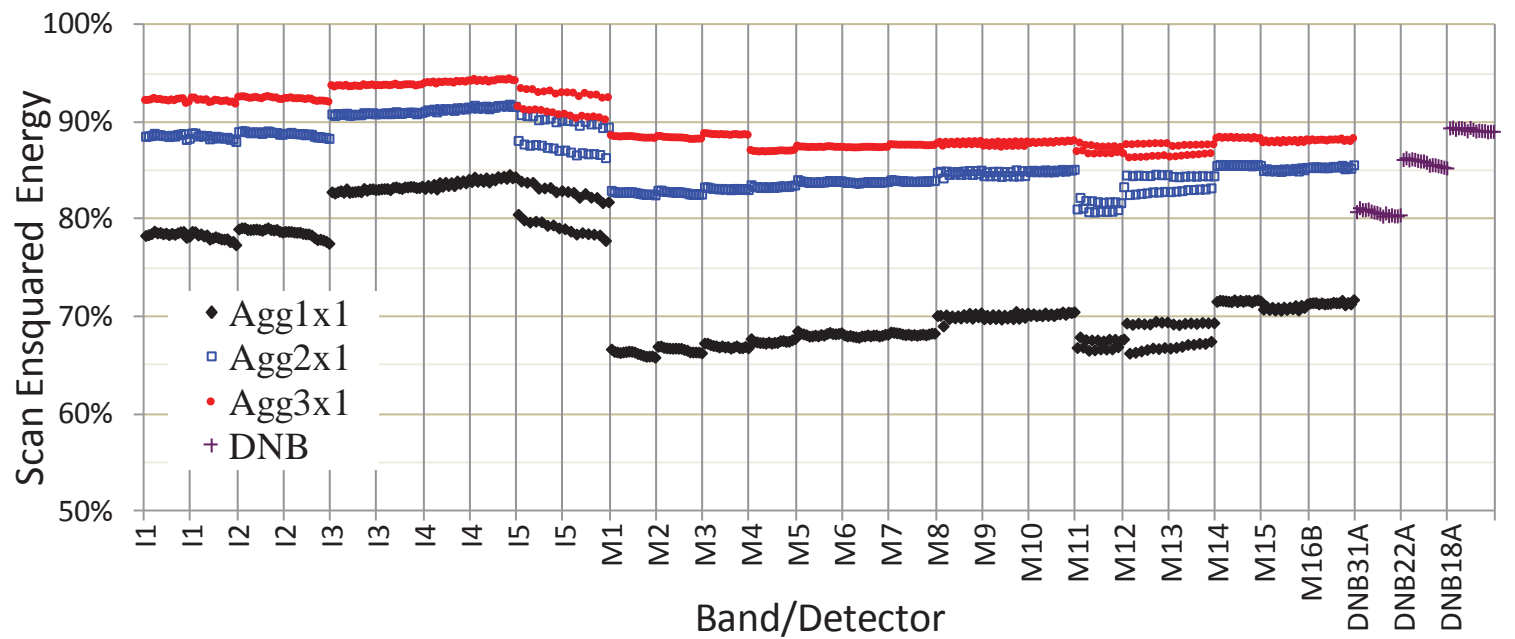

Figure 5. Scan direction Ensquared energy (EE) for the each detector for the I-bands, M-bands and DNB (HGA).

Figure 6 shows the scan direction integrated out-of-pixel (IOOP) spatial response for each detector for the I-bands and M-bands in the three aggregation zones, and three DNB (HGA) aggregation modes. This is the amount of energy contaminated by the neighboring pixels. As we can see, band M1 pixels are contaminated the most, up to 35\% from the neighboring pixels in the Agg1x1 zones. 


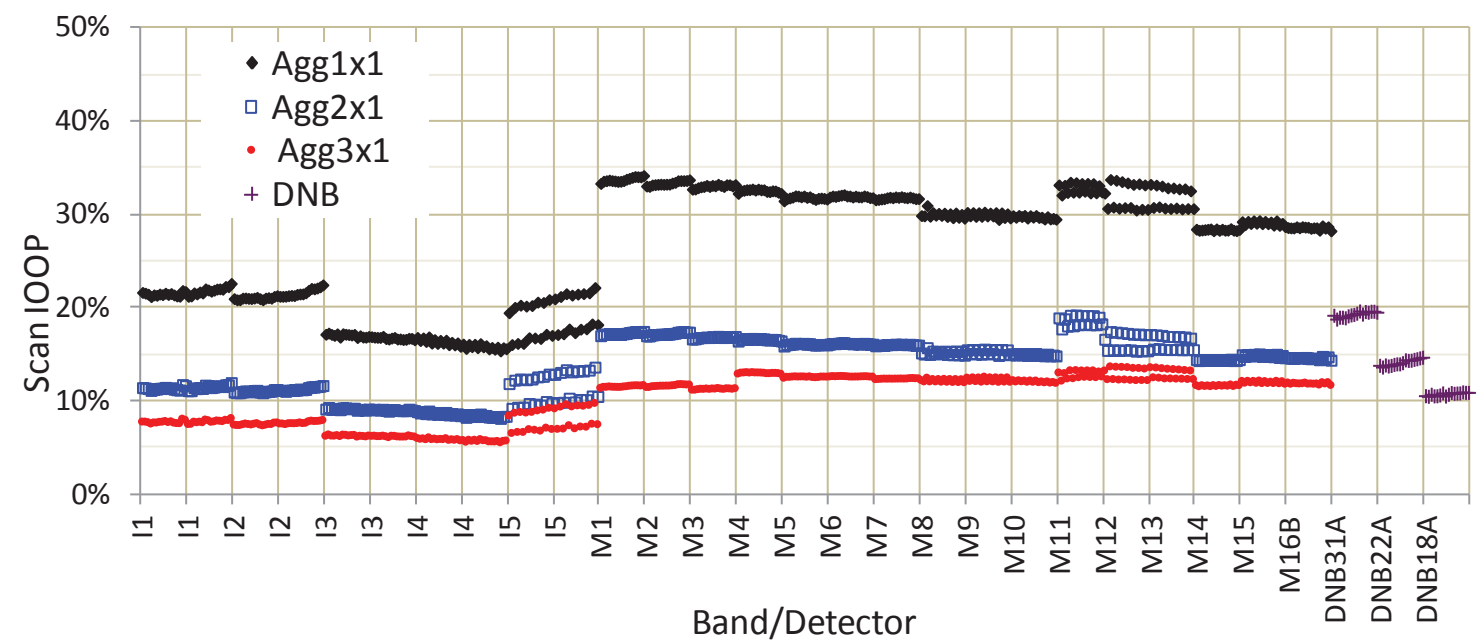

Figure 6. Scan direction Integrated Out-Of-Pixel (IOOP) spatial response for the each detector for the I-bands, Mbands and DNB (HGA).

\subsection{On-orbit spatial response characterization}

For on-orbit VIIRS bands spatial response characterization (for verification purposes), we devise an approach that takes advantage of an available high contrast linear feature to provide a direct, although approximate, measurement of the VIIRS instrument along-scan LSF. In our approach we use a co-geo located Landsat TM image to precisely locate the linear feature in the VIIRS image. We use the Lake Ponchartrain Causeway as a high contrast linear feature. Figures 7(a) and 7(b) show sections of Landsat TM and VIIRS images co-geolocated to a spatial resolution 13 times finer than the VIIRS image resolution (and slightly finer than the Landsat TM resolution). The data dropouts in the Landsat TM image are due to the well-known scan line corrector failure on Landsat $7^{[13]}$ (and are not included in the analysis). We locate the causeway in the Landsat TM image by processing the co-geolocated Landsat TM image with the HSeg ${ }^{[14]}$ image segmentation and selecting region objects corresponding to the causeway with HSegViewer utility. We then compute the linear best fit equation to the found causeway pixel locations as a precise model of the causeway location. A plot of this best fit linear best fit equation is shown as the red line in Figure 7(c). We also noted where the center of each VIIRS image pixel was located in relation to the modeled causeway location, shown as the cyan colored dots in Figure 7(c).
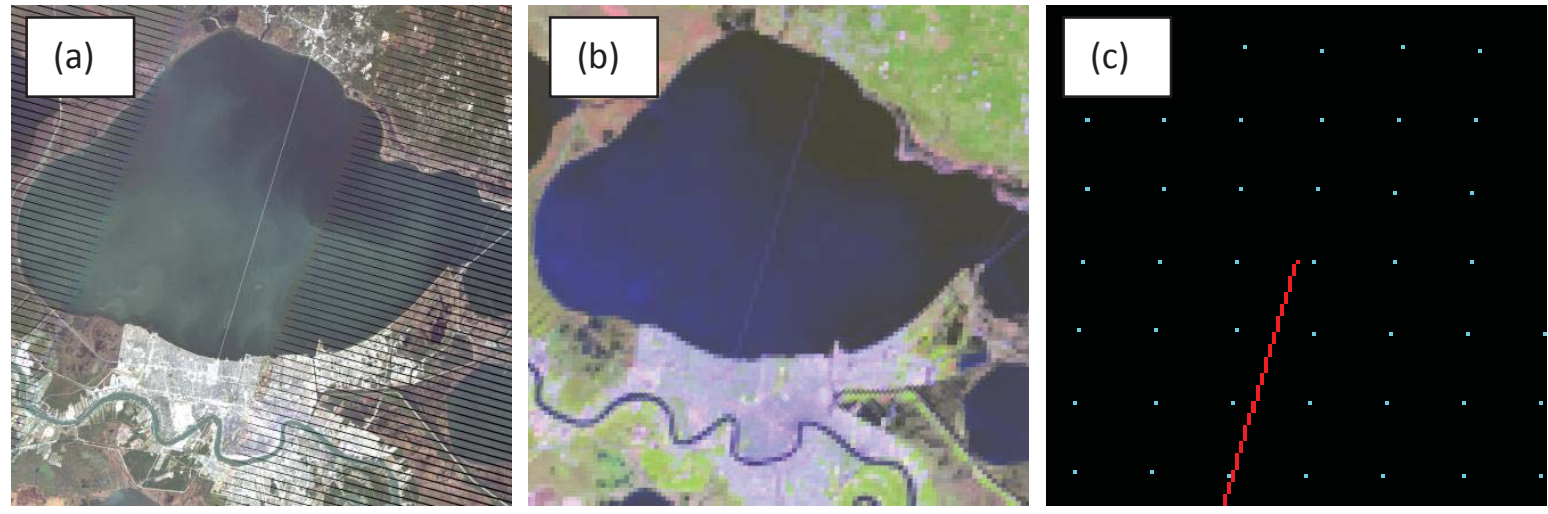

Figure 7. Co-geolocated Lake Ponchartrain subsets for (a) a Landsat TM image from 21 Nov. 2012 and (b) a VIIRS image from 18 Nov. 2012. (c) 16-times magnified plot of the linear equation fit to the northern end of the causeway (red line) and the locations of the centers of the SNPP VIIRS image data pixels (cyan dots).

We then used the information embodied in Figure 7(c) to find the fractional column causeway location in each scan line of the VIIRS image that crossed the causeway, and plotted the VIIRS radiance values of the 5 pixels in each scan line closest to the causeway. For this example in the Agg2x1 zone, we then fit these values to a sum of two identical 
Gaussians curves (each centered at +/- 0.5 HSI). The LSF measurements, model fit and pre-launch measurement are shown in Figure 8 for bands I2 and I3, the raw data points and the modeled curves tightly follow the pre-launch curves. For bands I1 and I4 there is a much larger scatter in the data points. The most scattered data points can be found in band I5. However, the modeled curves are still similar to the pre-launch curves.

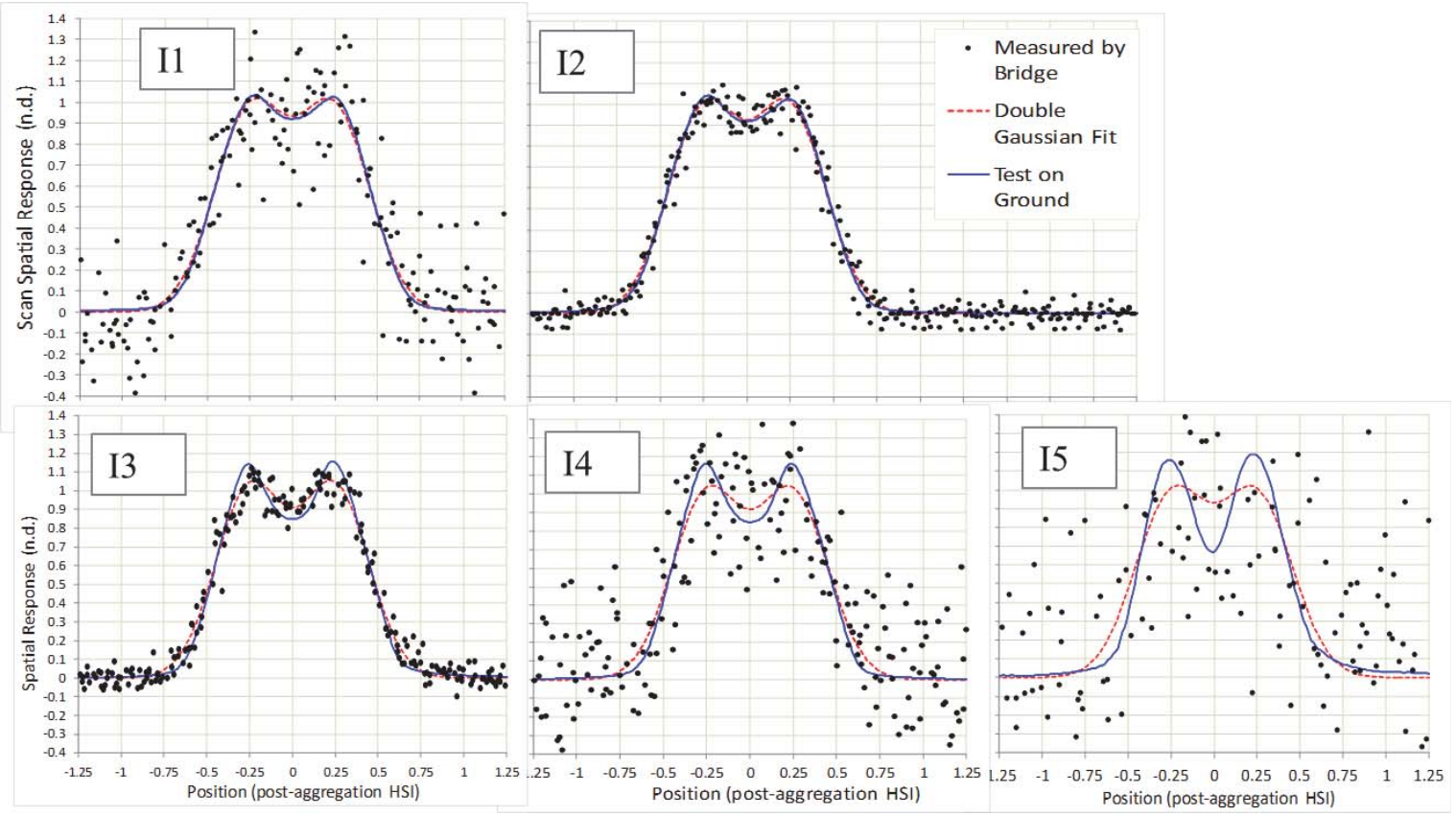

Figure 8. Scan direction LSFs for 5 I-bands in Agg2x1 zone, measured by the bridge, the double-Gaussian fit and the pre-launch data.

These modeled LSFs yield FWHM as the sensor GDFOVs for the 5 I-bands. The mean values and standard deviations binned in the three aggregation zones are plotted in Figure 9, along with pre-launch data. As we can see, the on-orbit LSFs measurements based on the ground target compare favorably with the pre-launch test, especially in the aggregation zones close to the start of scan, especially considering the atmospheric modulation ${ }^{[12]}$. For the GDFOV measured from the end of the scan, the sensor zenith angles are in the opposite direction from the solar zenith angles and so sun glint may affect the GDFOV retrievals. Further study of the effects of sun glint is on-going, along with the retrievals of GDFOVs for the M-bands and DNB.

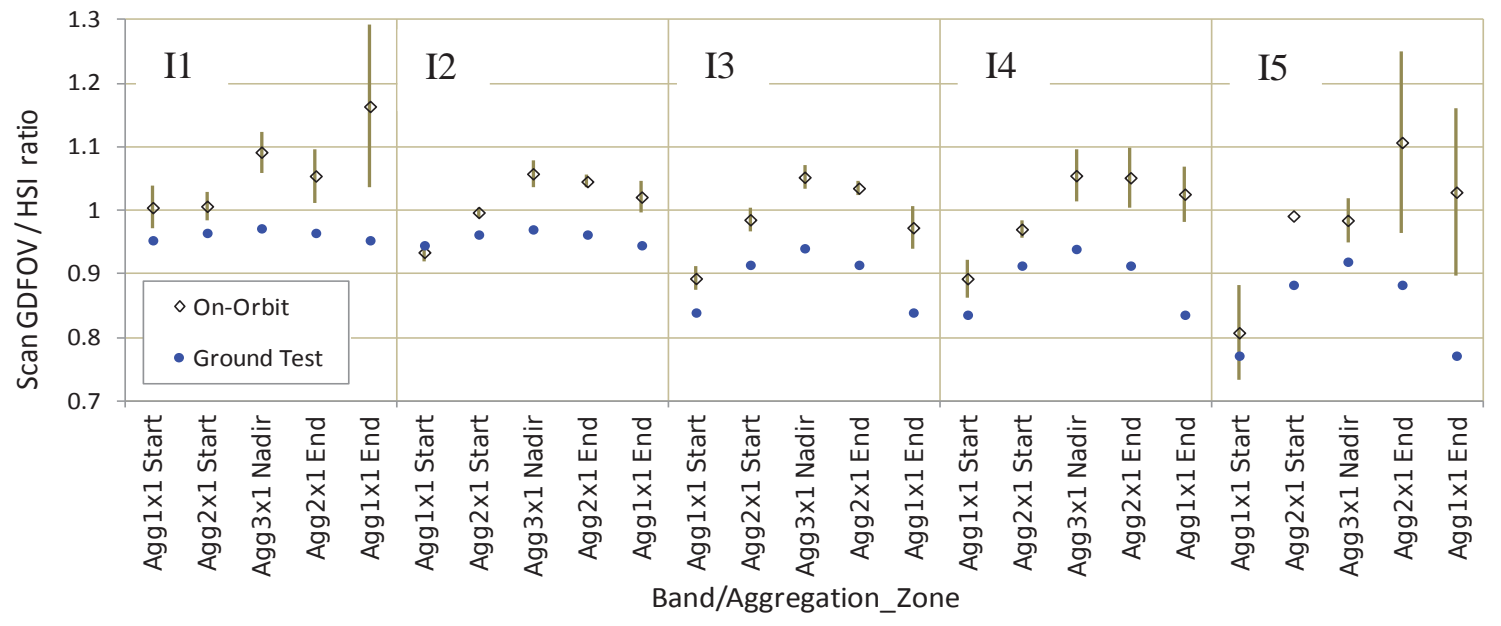

Figure 9. Scan direction GDFOV to HIS ratio for the 5 I-bands using Gaussian fit as shown in Figure 8 in the 5 aggregation zones from the start to end of scan. 


\section{BAND-TO-BAND CO-REGISTRATION (BBR)}

The BBR is a statistical value of a collection of individual detector-to-detector co-registration (DDR) measurments between two corresponding spectral bands. DDR may be defined as the overlap of point spread functions (PSFs) from one detector in one spectral band to a corresponding detector in another spectral band ${ }^{[4,15,16]}$.

$$
D D R_{i, j}=1-\oiint_{(x, y) \in R}\left|P S F_{i}-P S F_{j}\right| / 2 d x d y
$$

where $P S F$ is normalized, i.e.,

$$
\oiint_{(x, y) \in R} P S F d x d y=1
$$

Since the exact PSF is usually difficult to obtain precisely, especially for space-borne sensors which are subject to various conditions, an approximation of Equation (6) is used in practice. Measurements are done to estimate apparent relative locations (offsets) of detectors between corresponding band pairs. The offsets themselves are then be used as measures of BBR, as is done for the Moderate Resolution Imaging Spectroradiometer (MODIS) onboard NASA Earth Observing System (EOS) Terra and Aqua satellites ${ }^{[17,18,19,20]}$. For VIIRS, the offsets used to compute BBR matrices are based on a chosen reference area ${ }^{[4]}$ with the assumption that an equivalent PSF is evenly distributed over that area. This may be expressed as

$$
D D R_{i, j} \approx\left(1-\frac{\Delta S_{i, j}}{L_{S}}\right)\left(1-\frac{\Delta T_{i, j}}{L_{T}}\right)
$$

where $\Delta S_{i, j}$ and $\Delta T_{i, j}$ are the measured detector offsets (in HSI units) and $L_{S}$ and $L_{T}$ are the length scales approximating the equivalent area in the scan and track directions, respectively.

The VIIRS performance requirements specify the length scales at "specified HSR" at the ends of scan in both scan and track directions at $1600 \mathrm{~m}$ for M-bands and at 800 for I-bands. As we described in the beginning of Section 2, such length scales are comparable with the HSI at the ends of the scan. Thus, the HSI was used as the length scales to assess whether VIIRS met its BBR requirement ${ }^{[4]}$. In the following subsections, we are going to present the offsets measured pre-launch to gauge the "improvements" of BBR in the Agg2x1 and Agg3x1 zones relative to the Agg1x1 zone BBR using the HSI as defined in the VIIRS requirements. We will then compare this to the BBR from alternative approaches based on the scan direction length scales defined by the GDFOV and HSR. In this analysis, the length scale in the track direction is kept the same as track HSI. Since this paper focuses on the comparisons purpose, we ignore the effects of on-orbit conditions (e.g. atmosphere blurring).

\subsection{Band-to-band offsets}

Figure 10 shows the band averaged offsets relative to band I1 in the scan and track directions. Data for 2x1 and 3x1 aggregation zones are derived from measurements in thermal vacuum at the nominal temperature performance plateau in the diagnostic mode, i.e., Agg1x1 zone. The relative positions of all detectors were precisely measured ${ }^{[4]}$. We only show band averaged values here for clarity. 


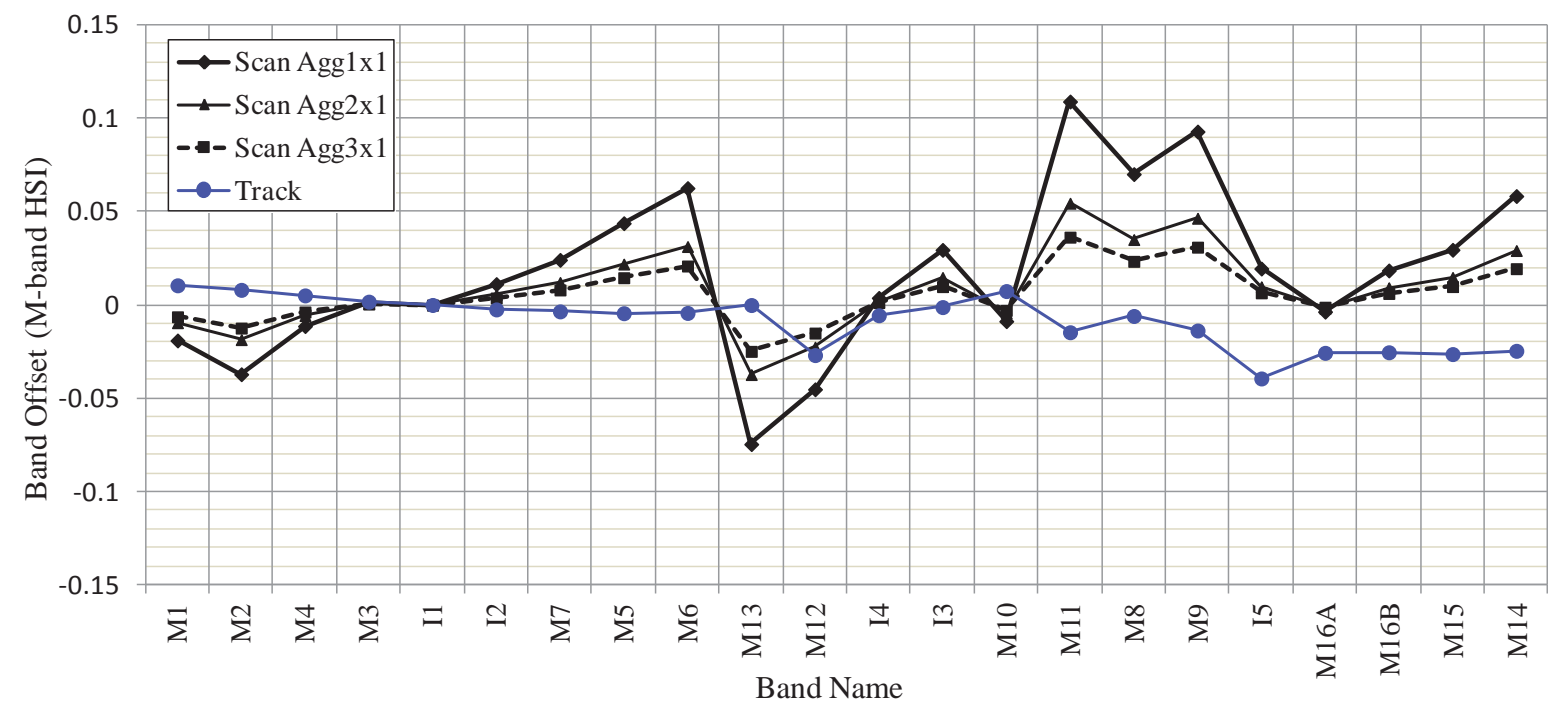

Figure 10. Band average offset from ground test under thermal vacuum condition at the nominal temperature performance plateau.

\subsection{BBR matrices based on HSI}

In the Agg1x1 zone the DDRs have been calculated based on the HSIs in the scan and track directions. Note, since these BBR results are for the Agg1x1 zone in which 4 (8) M-band (I-band) detectors are deleted on-board, these DDRs are based on the center 12 (24) M-band (I-band) detectors. Taking the minimum of the DDRs as the representation of BBR between the band pairs, the BBR matrix (in \%) for every band pair is presented in the upper-right triangle in Figure 11. The lower-left triangle is the VIIRS BBR specification for "at least $99.7 \%$ of corresponding pixel samples". To make the figures easier to understand, colors in the matrix indicate how well each band-pair is co-registered (with "green" the best and "pink" the worse), they are not meant to indicate compliance with the requirement. All VIIRS band-pairs met the requirement except pairs M9/M13 and M11/M13, which were only non-compliant by a small amount.

We then use the offsets in term of post-aggregation HSI and compute the BBR matrices in the Agg2x1 and Agg3x1 zones, as shown respectively in the upper-right and lower-left triangles in Figure 12. In this figure, we see the "improvements" of BBR up to 10\% and 13\% respectively in the Agg2x1 and Agg3x1 aggregation zones. 


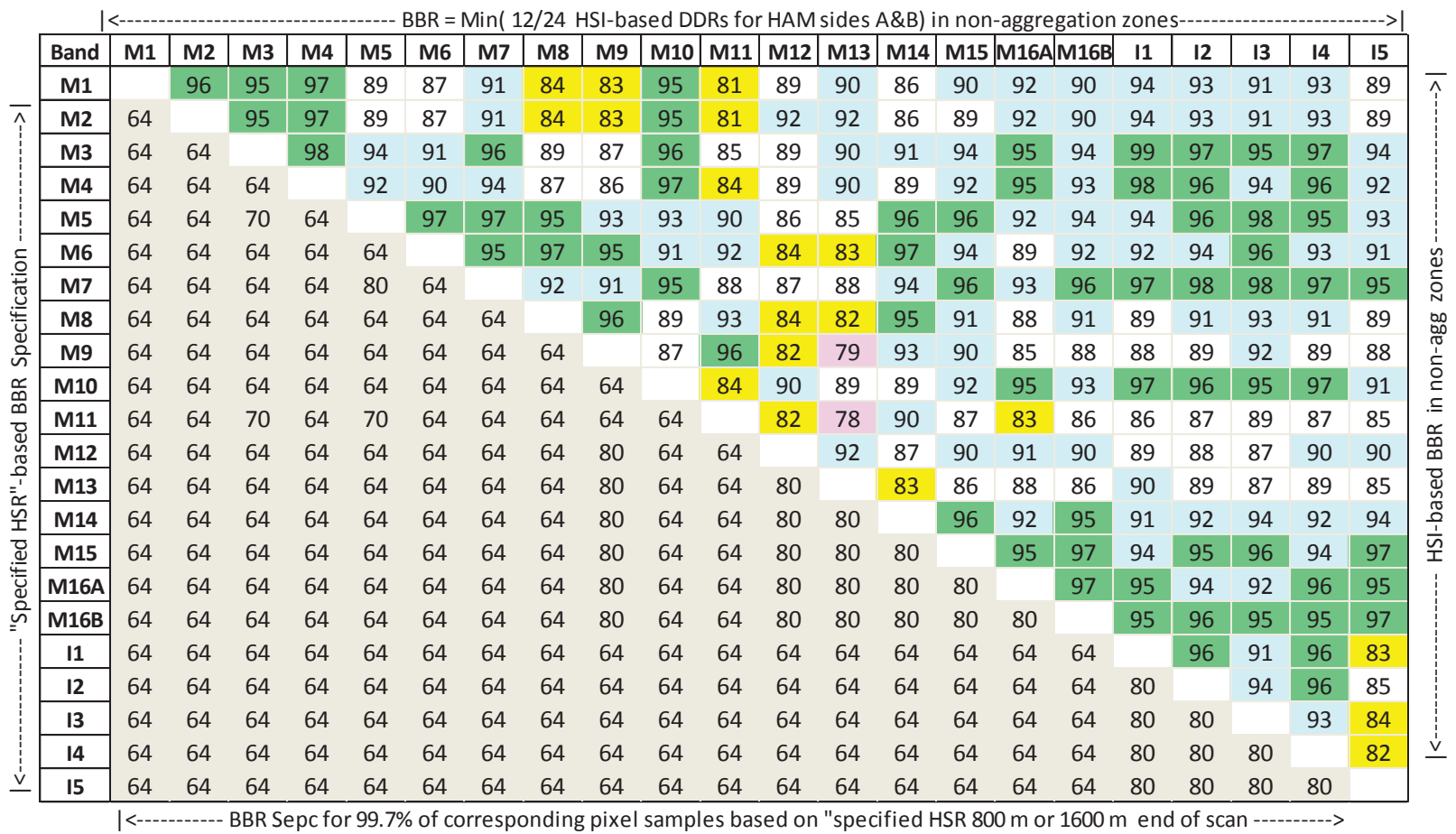

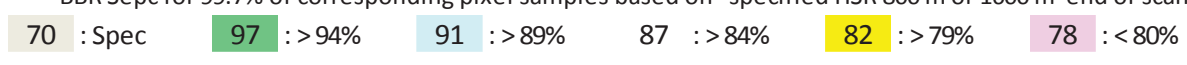

Figure 11 BBR based on HSI. Lower left triangle: specification at 99.7\% confidence level. Upper right triangle: minimum among detector pairs based on HSI in the Agg1x1 zones.

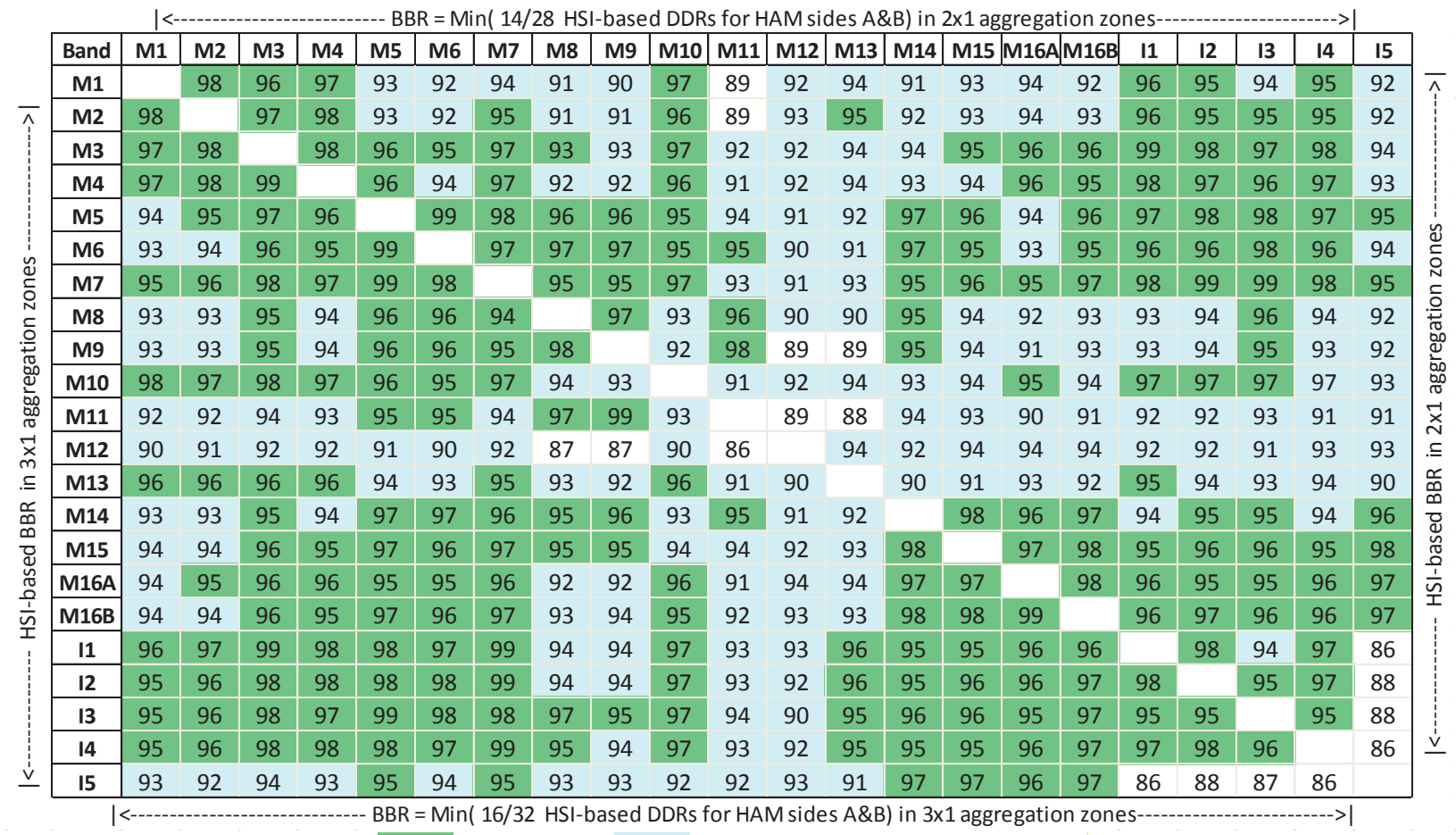

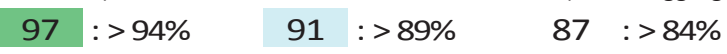

Figure 12 BBR based on the HIS in Agg2x1 zones (upper right triangle) and Agg3x1 zones (lower left triangle). 


\subsection{Alternative parameterization of BBR based on GDFOV and HSR}

As we shown in Figure 2 and Figure 3, the GDFOV and HSR in the scan direction are different from the HSI, especially in the Agg1x1 zones. As above, in this section we set the length scale to the HSI (which is about the same as $\mathrm{GIFOV}^{[4]}$ ), but change the scan direction length scale to GDFOV and HSR. The results are shown in Figure 13. Compared to the BBR matrix based on HSI, the BBR based on GDFOV "improves" (up to 4\%) for the M-band pairs but "degrades" (up to 3\%) for the I-band pairs, while BBR based on scan HSR "improves" the M-band pairs (up to 4\%).

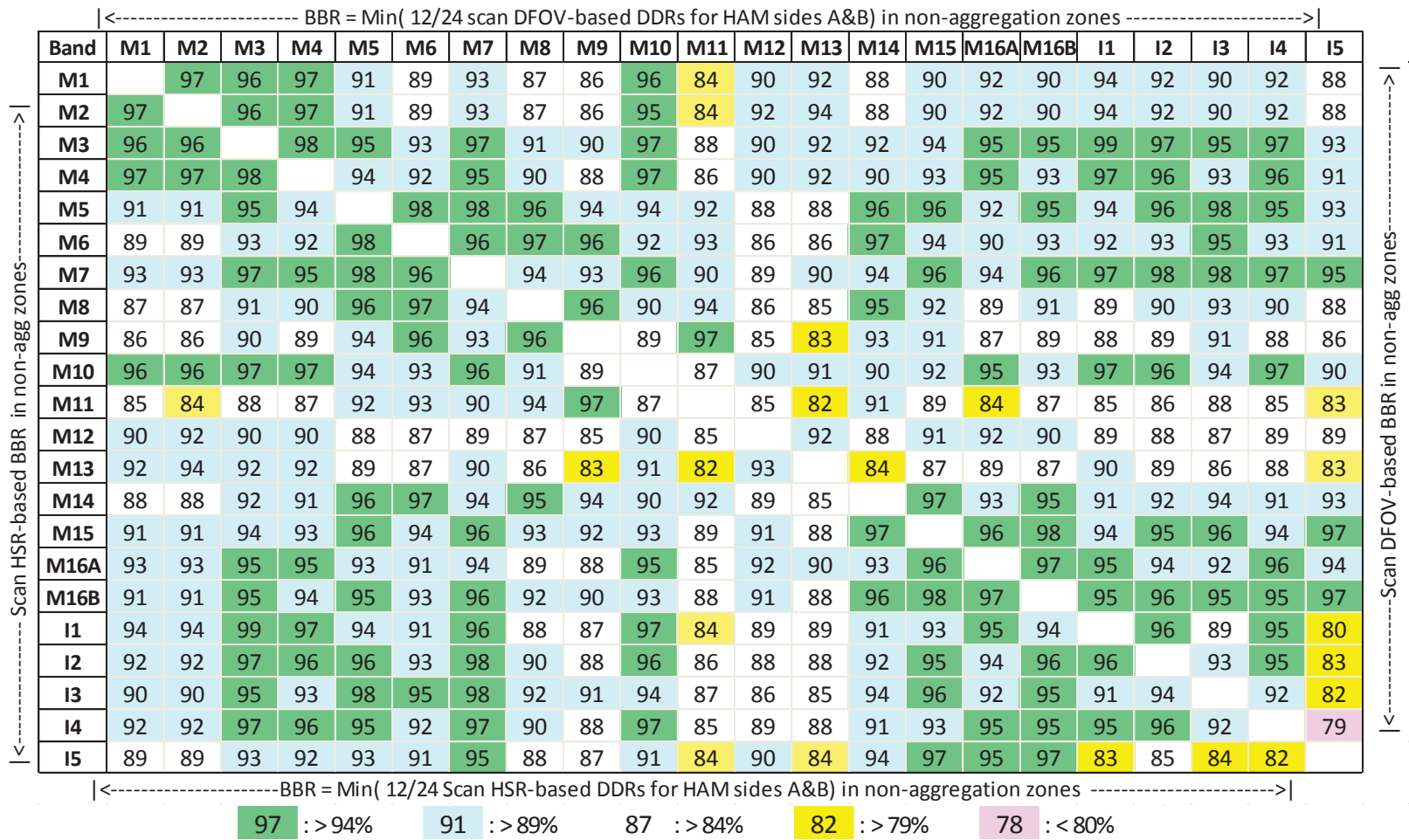

Figure 13 BBR matrices based on scan GDFOV (upper right) and scan HSR (lower left).

To be consistent, when we use the scan HSR for the length scale in the scan direction, we should use track HSR for the length scale (which is close to the theoretical value of $0.83 \mathrm{GIFOV}^{[4]}$ ) for the track direction as well. This is done and the resultant BBR matrix is compared with the BBR matrix based on HSI (Figure 14). Using HSR as the base for both the scan and track direction, the BBR "improves" (up to 4\%) for the M-band pairs but "degrades" (up to 3\%) for the Iband pairs. Using HSR as the length scale for nearly square LSFs, the offset between the band pairs are amplified $20 \%$. For the case of SNPP, the offsets in the track direction are relatively small (Figure 10) and thus the amplification is small. If the offsets are relatively large (similar to scan offsets in Agg1x1 zone as shown in Figure 10), then, that 20\% amplification could eat away the small BBR margin. 


\begin{tabular}{|c|c|c|c|c|c|c|c|c|c|c|c|c|c|c|c|c|c|c|c|c|c|c|c|}
\hline & Band & M1 & M2 & M3 & M4 & M5 & M6 & M7 & M8 & M9 & \begin{tabular}{|l|} 
M10 \\
\end{tabular} & \begin{tabular}{|l|} 
M11 \\
\end{tabular} & M12 & M13 & \begin{tabular}{|l|} 
M14 \\
\end{tabular} & M15 & M16A & M16B & I1 & 12 & 13 & 14 & 15 \\
\hline & M1 & & 96 & 95 & 97 & 89 & 87 & 91 & 84 & 83 & 95 & 81 & 89 & 90 & 86 & 90 & 92 & 90 & 94 & 93 & 91 & 93 & 89 \\
\hline & M2 & 97 & & 95 & 97 & 89 & 87 & 91 & 84 & 83 & 95 & 81 & 92 & 92 & 86 & 89 & 92 & 90 & 94 & 93 & 91 & 93 & 89 \\
\hline & M3 & 96 & 96 & & 98 & 94 & 91 & 96 & 89 & 87 & 96 & 85 & 89 & 90 & 91 & 94 & 95 & 94 & 99 & 97 & 95 & 97 & 94 \\
\hline & M4 & 97 & 97 & 98 & & 92 & 90 & 94 & 87 & 86 & 97 & 84 & 89 & 90 & 89 & 92 & 95 & 93 & 98 & 96 & 94 & 96 & 92 \\
\hline & M5 & 91 & 91 & 95 & 94 & & 97 & 97 & 95 & 93 & 93 & 90 & 86 & 85 & 96 & 96 & 92 & 94 & 94 & 96 & 98 & 95 & 93 \\
\hline & M6 & 89 & 89 & 93 & 92 & 98 & & 95 & 97 & 95 & 91 & 92 & 84 & 83 & 97 & 94 & 89 & 92 & 92 & 94 & 96 & 93 & 91 \\
\hline & M7 & 92 & 92 & 96 & 95 & 98 & 96 & & 92 & 91 & 95 & 88 & 87 & 88 & 94 & 96 & 93 & 96 & 97 & 98 & 98 & 97 & 95 \\
\hline & M8 & 87 & 87 & 90 & 90 & 95 & 97 & 94 & & 96 & 89 & 93 & 84 & 82 & 95 & 91 & 88 & 91 & 89 & 91 & 93 & 91 & 89 \\
\hline & M9 & 86 & 86 & 90 & 89 & 94 & 96 & 93 & 96 & & 87 & 96 & 82 & 79 & 93 & 90 & 85 & 88 & 88 & 89 & 92 & 89 & 88 \\
\hline & M10 & 96 & 95 & 97 & 97 & 93 & 92 & 95 & 90 & 88 & & 84 & 90 & 89 & 89 & 92 & 95 & 93 & 97 & 96 & 95 & 97 & 91 \\
\hline & M11 & 84 & 84 & 88 & 86 & 91 & 93 & 90 & 94 & 97 & 87 & & 82 & 78 & 90 & 87 & 83 & 86 & 86 & 87 & 89 & 87 & 85 \\
\hline & M12 & 89 & 91 & 89 & 89 & 87 & 86 & 88 & 86 & 85 & 89 & 85 & & 92 & 87 & 90 & 91 & 90 & 89 & 88 & 87 & 90 & 90 \\
\hline & M13 & 92 & 93 & 92 & 92 & 88 & 86 & 90 & 86 & 83 & 91 & 82 & 92 & & 83 & 86 & 88 & 86 & 90 & 89 & 87 & 89 & 85 \\
\hline & M14 & 87 & 88 & 92 & 90 & 96 & 97 & 94 & 95 & 93 & 90 & 91 & 88 & 85 & & 96 & 92 & 95 & 91 & 92 & 94 & 92 & 94 \\
\hline & M15 & 90 & 90 & 94 & 92 & 95 & 94 & 96 & 92 & 91 & 92 & 89 & 91 & 87 & 97 & & 95 & 97 & 94 & 95 & 96 & 94 & 97 \\
\hline & M16A & 92 & 92 & 94 & 95 & 92 & 90 & 93 & 89 & 87 & 94 & 85 & 92 & 89 & 93 & 96 & & 97 & 95 & 94 & 92 & 96 & 95 \\
\hline & M16B & 90 & 90 & 94 & 93 & 95 & 93 & 96 & 91 & 90 & 92 & 87 & 91 & 88 & 96 & 98 & 97 & & 95 & 96 & 95 & 95 & 97 \\
\hline & 11 & 94 & 94 & 99 & 97 & 94 & 91 & 96 & 88 & 87 & 97 & 84 & 88 & 89 & 90 & 93 & 94 & 94 & & 96 & 91 & 96 & 83 \\
\hline & 12 & 92 & 92 & 97 & 95 & 96 & 93 & 98 & 90 & 88 & 96 & 85 & 87 & 88 & 91 & 94 & 93 & 95 & 96 & & 94 & 96 & 85 \\
\hline & 13 & 90 & 89 & 95 & 93 & 97 & 95 & 98 & 92 & 91 & 94 & 87 & 86 & 85 & 93 & 95 & 91 & 94 & 91 & 94 & & 93 & 84 \\
\hline & 14 & 92 & 92 & 97 & 95 & 94 & 92 & 97 & 90 & 88 & 96 & 85 & 88 & 87 & 90 & 93 & 95 & 94 & 95 & 95 & 92 & & 82 \\
\hline & 15 & 88 & 88 & 93 & 91 & 93 & 90 & 94 & 88 & 86 & 89 & 83 & 89 & 84 & 93 & 97 & 94 & 96 & 81 & 83 & 82 & 80 & \\
\hline
\end{tabular}

Figure 14 BBR matrices based on HSI (upper right) and scan/track HSR (lower left).

In theory, for an ideal triangular LSF (which is a close approximation of scan $\operatorname{LSF}^{[5,17,19,20]}$ ) with an FWHM the same as its ASI and GDFOV the same as its HSI, the HSR is 1.13 HSI, 13\% larger than the footprint usually represented by the FWHM. For an ideal square LSF (which is a close approximation of track LSF ${ }^{[4,5,18,19,20]}$ ) with an FWHM the same as its ASI and GIFOV the same as its HSI, the HSR is $0.83 \mathrm{HSI}, 17 \%$ smaller than the footprint usually represented by the FWHM. HSR is calculated as the half wave-length where modulation transfer function (MTF) of LSF drops to $1 \frac{1}{2}$, i.e., the contrast of a sinusoidal wave at that wave-length is reduced to $1 / 2$. This is sometime called effective resolution. It lacks the general meaning of representing the size of the PSF or LSF patterns projected on the ground. It could be misleading to use HSR as a measure of image quality or as a length scale to calculate BBR. Therefore, HSR as defined in Equation (3) should not be used in the field of remote sensing, especially when the remote sensed data is used to further retrieve biogeophysical parameters.

\section{CONCLUDING REMARKS}

In this paper, we present the parameterization of sensor spatial responses and band-to-band co-registration (BBR) in various ways. These results are derived from recently re-processed data measured before the SNPP VIIRS was launched in October 2011. For the VIIRS spatial response in the scan direction, all 5 I-bands are under-sampled as measured by their ground dynamic field of view (GDFOV). The image quality as measured by modulation transfer function (MTF), ensquared energy (EE) and integrated out-of-pixel (IOOP) spatial response are high. However, undersampling may cause aliasing. For the $16 \mathrm{M}$-bands, GDFOV values in the scan direction indicated that pixels in the nonaggregated zones near the edges of scan are over-sampled, their image quality measured by MTF is relatively low (compared to I-bands) and contamination from neighboring pixels as indicated by IOOP is relatively high (compared to I-bands). In the Agg2x1 and Agg3x1 zones, M-bands pixels are sampled to the Nyquist frequency. In terms of BBR, all band pairs have offsets less than $20 \%$ of their corresponding pixel sizes. They have near $80 \%$ or better footprint overlaps, especially in the Agg2x1 and Agg3x1 zones. When comparing HSI, GDFOV and HSR as the length scales for fractional band-to-band mis-registration, we found that GDFOV calculated as the full-width at half-maximum (FWHM) 
of sensor spatial response function is a better representation of footprint size. We also propose that the HSR as defined in Equation (3) is neither an adequate representation of the size of sensor spatial response nor an adequate measure of imaging quality, and therefore HSR concept should not be used in the field of remote sensing when the remote sensed data is used to retrieve biogeophysical parameters.

\section{ACKNOWLEDGEMENTS}

The authors acknowledge the sponsorship of this work by the SNPP SDS, the SNPP Project Science Office and NOAA Center for Satellite Applications and Research (STAR), and the cooperation and assistance from many colleagues at The Aerospace Corporation, Raytheon Company, Northrop Grumman Aerospace Systems, the SNPP Land PEATE, the VIIRS Calibration Support Team (VCST) Radiometric Group, and the JPSS Program Office. The manuscript contents are solely the opinions of the authors and do not constitute a statement of policy, decision, or position on behalf of Innovim, Sigma Space Corporation, NASA or the U.S. government.

\section{REFERENCES}

[1] Cao, C., F. DeLuccia, X. Xiong, R. Wolfe, F. Weng (2013), "Early On-orbit Performance of the Visible Infrared Imaging Radiometer Suite (VIIRS) onboard the Suomi National Polar-orbiting Partnership (S-NPP) Satellite," IEEE Trans. on Geosci. and Remote Sens., in press, DOI:10,1109/TGRS.2013.2247768.

[2] Schueler, C., J. E. Clement, P. Ardanuy, C.Welsch, F. DeLuccia, and H. Swenson4 (2001), "NPOESS VIIRS Sensor Design Overview", Earth Observing Systems VI, William L. Barnes, Ed., Proceedings of SPIE Vol. 4483.

[3] Scalione, T., F. DeLuccia, J. Cymerman, E. Johnson, J. K. McCarthy and D. Olejnicza (2005), "VIIRS Initial Performance Verification -- Subassembly, Early Integration and Ambient Phase I Testing of EDU" 2005 IEEE International Geoscience and Remote Sensing Symposium Proceedings (25th) (IGARSS 2005) Held in Seoul, Korea on 25-29 July 2005.

[4] Lin, G., R. E. Wolfe, M. Nishihama (2011), "NPP VIIRS Geometric Performance Status," Earth Observing Systems XVI, edited by James J. Butler, Xiaoxiong Xiong, Xingfa Gu, Proc. of SPIE, Vol. 8153, pp. 81531V81531V-14, doi: 10.1117/12.894652, Sept. 2011.

[5] Wolfe, R.E., G. Lin, M.Nishihama, K.P. Tewari, E. Montano (2012), "NPP VIIRS Early On-Orbit Geometric Performance", Earth Observing Systems XVII, edited by J. J. Butler, X. Xiong, X. Gu, Proc. of SPIE Vol. 8510, 851013, doi: 10.1117/12.929925, Oct. 2012.

[6] Cao, C., X. Xiong, R. Wolfe, F. Deluccia, Q. Liu, S. Blonski, G. Lin, M., Nishihama, D. Pogorzala, and H. Oudrari (2013). "Visible/Infrared Imager Radiometer Suite (VIIRS) Sensor Data Record (SDR) User's Guide," U.S. Dept. Commerce, NOAA, Silver Spring, MD, USA, Tech. Rep. NESDIS 142, Ver. 2.1, April 2013.

[7] Wolfe, R.E., G. Lin, M. Nishihama, K. P. Tewari, J. C. Tilton, A. R. Isaacman (2013), "Suomi NPP VIIRS prelaunch and on-orbit geometric calibration and characterization", Journal of Geophysical Research Atmospheres, accepted.

[8] Fang, D.T and J. Puschell (2010), "Imagery spatial performance throughput correction methodology," Remote Sensing System Engineering III, Proc. of SPIE, 7813, doi: 10.1117/12.860740.

[9] Schowengerdt, R. (2006), Remote Sensing -- Models and Methods for Image Processing, Academic Press, $3^{\text {rd }}$ Edition, 2006. ISBN: 978-0-12-369407-2. 560pp.

[10] Oppenheim, A.V., R. W. Schafer (1989), Discrete-time signal processing, Prentice Hall, 1989, 879 pp.

[11] Zhang, Peng, J.Li, E.Olson, T. J. Schmit, J.Li, and W. P.Menzel (2006), "Impact of Point Spread Function on Infrared Radiances From Geostationary Satellites", TRANSACTIONS ON GEOSCIENCE AND REMOTE SENSING, VOL. 44, NO. 8, pp 2176-2183, AUGUST 2006.

[12] Buskila, K., S. Towito, E. Shmuel, R. Levi, N. Kopeika, K. Krapels, R. G. Driggers, R. H. Vollmerhausen, and C. E. Halford (2004), "Atmospheric modulation transfer function in the infrared," APPLIED OPTICS, Vol. 43, No. 2, pp471-482.

[13] Storey, J., P. Scaramuzza, G. Schmidt, and J. Barsi (2005), "Landsat 7 Scan Line Corrector-off Gap-filled Product Development," in Proceedings of Pecora 16, Global Priorities in Land Remote Sensing, October 23-27, 2005, Sioux Falls, South Dakota. 
[14] Tilton, J. C., Y. Tarabalka, P. M. Montesano, and E. Gofman (2012), "Best Merge Region Growing Segmentation with Integrated Non-Adjacent Region Object Aggregation," IEEE Transactions on Geoscience and Remote Sensing, vol. 50, no. 11, pp. 4454-4467, Nov. 2012.

[15] Skauli, Torbjørn (2011), "Quantifying coregistration errors in spectral imaging", Imaging Spectrometry XVI, edited by Sylvia S. Shen, Paul E. Lewis, Proc. of SPIE Vol. 8158, 81580A, doi: 10.1117/12.894699., Sept., 2011.

[16] Skauli, Torbjørn (2012), "An upper-bound metric for characterizing spectral and spatial coregistration errors in spectral imaging," Optics Express, Vol. 20, Issue 2, pp. 918-933 (2012).

[17] Montgomery, H., N. Che, and J. Bowser, "Determination of the spatial characteristic by using the SpectroRadiometric Calibration Assembly (SRCA) of MODIS (Part I. Along-scan)”, Proc. of SPIE, Vol. 3439, San Diego, 1998, pp.226-237.

[18] Montgomery, H., N. Che, and J. Bowser, "Determination of the spatial characteristic by using the SpectroRadiometric Calibration Assembly (SRCA) of MODIS (Part II. Along-track)", Proc. of SPIE, Vol. 3439, San Diego, 1998, pp.238-246

[19] Xiong, X., N.Che, and W. Barnes, "Terra MODIS On-Orbit Spatial Characterization and Performance", IEEE Trans. Geo. \& Remote Sens., Vol. 43, pp. 355-365, 2005.

[20] Xiong, X., N. Che, W. Barnes, Y. Xie, L. Want, and J. Wu, "Status of Aqua MODIS Spatial Characterization and Performance", Proc. of SPIE, Vol. 6361, doi: 10.1117/12.687162, 2006. 